\title{
Discovering Groups of People in Google News
}

\author{
Dhiraj Joshi \\ Department of Computer Science and \\ Engineering \\ The Pennsylvania State University \\ University Park, PA-16802, USA \\ djoshi@cse.psu.edu
}

\author{
Daniel Gatica-Perez \\ IDIAP Research Institute and Ecole \\ Polytechnique Federale de Lausanne (EPFL) \\ $\mathrm{CH}-1920$, Martigny, Switzerland \\ gatica@idiap.ch
}

\begin{abstract}
In this paper, we study the problem of content-based social network discovery among people who frequently appear in world news. Google news is used as the source of data. We describe a probabilistic framework for associating people with groups. A low-dimensional topic-based representation is first obtained for news stories via probabilistic latent semantic analysis (PLSA). This is followed by construction of semantic groups by clustering such representations. Unlike many existing social network analysis approaches, which discover groups based only on binary relations (e.g. co-occurrence of people in a news article), our model clusters people using their topic distribution, which introduces contextual information in the group formation process (e.g. some people belong to several groups depending on the specific subject). The model has been used to study evolution of people with respect to topics over time. We also illustrate the advantages of our approach over a simple co-occurrence-based social network extraction method.

Categories and Subject Descriptors: H.3.1 [Content Analysis and Indexing]: abstracting methods, linguistic processing.
\end{abstract}

General Terms: Algorithms, Experimentation, Human Factors.

Keywords: Text mining, social network analysis, probabilistic latent semantic indexing, topic evolution.

\section{INTRODUCTION}

Today technology plays a dual role of enhancing as well as understanding human life. Computers have revolutionized human interaction and redefined the meaning of staying in touch. New age communication entails exchange of large amounts of multimodal information generated by a host of devices at all times of the day. As a result, the popularity of gizmos like camera phones and the growth of the internet have created new challenges for data miners who wish to study and uncover human connectivity networks.

Permission to make digital or hard copies of all or part of this work for personal or classroom use is granted without fee provided that copies are not made or distributed for profit or commercial advantage and that copies bear this notice and the full citation on the first page. To copy otherwise, to republish, to post on servers or to redistribute to lists, requires prior specific permission and/or a fee.

HCM'06, October 27, 2006, Santa Barbara, California, USA.

Copyright 2006 ACM 1-59593-500-2/06/0010 ...\$5.00.
It has been argued that camera phones have changed the way personal photography has been perceived, in the past [19]. Search engines like Google index billions of pages containing text, images, and video. Information as diverse as huge picture galleries to authoritative news articles are at the click of a mouse. Hyperlink structure of the Web represents yet another important network between people, places and organizations which search engines like Google use to assign authority to Webpages. Network characteristics of the WWW have been carefully studied [3]. A more human oriented work addresses the similarities and differences between sociability and usability in context of online communities [17]. Approaches to build a web of trust in social aspects have been proposed [7]. The need for socially aware computation has been emphasized [16].

Besides being a massive repository of information, the WWW hides another form of social network between people which link analysis may often fail to uncover. Consider the travel blogs of two photographers who visit similar places and have an interest in photography but who may never know about each other due to their disjoint virtual spaces. Such connections will inevitably be missed by social network discovery methods which rely on direct contact between people such as email exchange. In our work, we propose a computational approach to model world news content to reveal one such social network between key players in global news events.

The automatic discovery of groups of people and their relations is an area of research that, although studied in social network analysis for several years [21], has seen a sharp increase of interest given the existence- and in some cases, public availability- of large amounts of data exchanged via e-mails [9], and posted on professional websites, chat rooms, blogs, etc., from which social connectivity patterns can be extracted [12]. An important source of information about people and their connections is Web news. In particular, Google news ${ }^{1}$ has become one of the richest access points to international news in terms of content and coverage (Fig. 1). Everyday this page displays representative text, pictures, and links to news stories deemed as most relevant by Google (Fig. 1). Links to stories from around 4500 international news sources can be obtained from here. Such an enormous coverage brings the advantages of providing complex multimedia content, and a better balance in terms of viewpoints (e.g. political and religious) across sources, which contrasts with existing

\footnotetext{
${ }^{1}$ http://news.google.com
} 
datasets widely used in text analysis (e.g. the text-only Reuters-21578 collection composed of newswires from a single news source [10]).

The goal of this work is to discover and quantify the emerging social network among people who occur frequently in news, by quantifying similarities between them in the context in which they appear in news. Many existing approaches for group discovery rely on the assumption that people's connections are described by simple binary relations (i.e. a pair of people are either related or not) [14]. In contrast, we aim at discovering the group structure of people in news using not only their co-occurrence in the same document, but also the document content itself. We present a simple model that first discovers the topic structure of a news collection, and then finds groups of people according to the discovered topics. The use of language information to detect relations between people using probabilistic models is an emergent trend $[11,20]$ that has been largely motivated by the recent invention of probabilistic models for collections of discrete data $[8,5]$. In a related work, an analysis of a social network emerging in news was recently reported in [15], with a different goal than ours, as it addressed the questions of whether the small-world and power-law degree-distribution properties - phenomena that have been recently observed in many complex networks [2] - appeared in social networks built from news articles. Importantly, the construction of the social network in [15] used binary co-occurrence information, not content as proposed here. In a different research line, multimedia news data has been used to learn correspondences between faces in images and names in photo captions [4]. In yet another work, multimedia sensors have been used to capture and study social interactions between people [6].

To the best of our knowledge, our work is one of the first studies on content-based discovery of social connectivity patterns using Web news data. Although our ultimate objective is to exploit social connectivity information existing in more than one media type (e.g. text and images), the approach presented here uses only textual information. Clearly, text is expected to constitute the strongest indicator of social relations in news, and the media type from which this information can be extracted more reliably. Later in the paper, we have discussed extension of the proposed approach to incorporate image information. A useful application of our work could be to discover soft links between travelers and photographers who maintain travel blogs containing text descriptions and pictures. Social network discovery using multimedia content can potentially reveal like minded people for future collaboration.

The remainder of the paper is organized in follows. In Section 2, we describe the data collection process and the nature of data collected. Section 3 describes our model to discover groups of people. In Section 4, we present the results and discussions. Section 5 provides some concluding remarks and discusses open issues.

\section{NEWS COLLECTION}

\subsection{News collection engine}

The goal of our news collection engine is to begin from the Google world news page and collect news articles from links available on that page. Google automatically categorizes news stories, and the 20 most relevant stories are presented with some representative text, images and links to similar stories. Similar stories are potentially versions of the same event or closely related stories, as reported by other news agencies. Extracting hyperlinks from HTML pages involves some basic parsing operations. For simplicity, we implemented these procedures on our own. The news collection engine performed the following tasks:

1. From Google's world news page, text for each of the top 20 news daily stories were identified and stored.

2. Google provides links to the current available instances of each of the 20 news events. An instance corresponds to a version of the story or closely related news reported by a news agency. Our program obtained each available version. These were stored in raw HTML format. On average, around 1500 to 2000 news articles were collected and stored everyday by our crawler. Some of these could be copies of old news articles. The duplicates were later identified and removed, leaving a smaller set of documents for further processing.

\subsection{News processing}

A public-domain Java-based HTML parser ${ }^{2}$ was used to extract text from HTML news files. News stories, collected over a period of about three weeks, formed our initial corpus for identifying an appropriate vocabulary to describe world news. Stopwords were identified and eliminated, stemming was performed, and words in news articles were ranked based on their frequency of occurrence to construct our vocabulary of 7413 words.

Additionally, we identified certain people occurring frequently in news. By observation, we discovered that proper nouns in text can be characterized by sequences of capital words (word beginning with an upper-case alphabet) followed by a lower case word. This characterization encompasses names of places, people, and news agencies. Therefore, a way of obtaining a list of people frequently occurring in news can be to track and obtain the frequencies of such sequences. Alternatively, one can also use a namedentity extraction program for the purpose. The obtained set was later manually trimmed to settle down upon a set of 32 people. The identified group of people, shown in Table 3, consists of politicians, terrorists, and heads of state of several nations, who appeared frequently in news between July 10, 2005 and July 31, 2005. As can be guessed from the list, the topics of interest during those weeks included the London bombings, the Israel-Palestine conflict, North Korea's nuclear program, Phillipines's political turmoil, etc. There are a few instances of the same person being spelt differently (e.g., Kim Jongil and Kim Jong Il), due to the lack of consistent naming among news agencies. In such cases, we treated differently spelt names, referring to the same person as different people.

\section{FINDING TOPICS AND GROUPS}

The algorithm we present here consists of two stages. Each news story from the collection is considered as a document and represented by a bag-of-words [1]. In the first stage of the algorithm, a topic-based representation is automatically learned from the news collection. In the second stage, groups of people are automatically found using the topic-based representation for each of the documents in

\footnotetext{
${ }^{2}$ http://htmlparser.sourceforge.net
} 




Figure 1: A snapshot of Google World News Page.

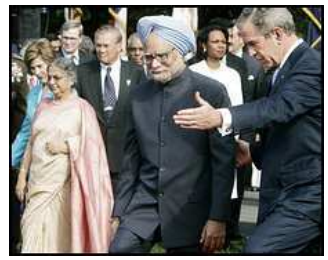

(a)

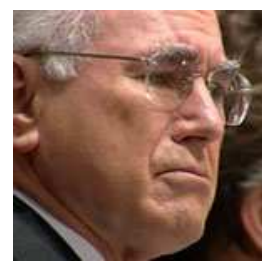

(b)

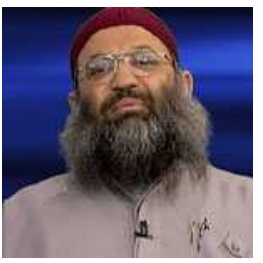

(c)

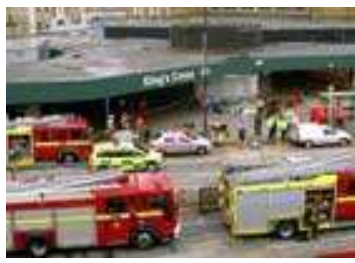

(d)

Figure 2: Four pictures obtained from Google news during the analyzed time period. The respective captions are (a) "Singh is escorted by Bush"; (b) "John Howard is standing by his criticism of a Melbourne cleric"; (c) "Islamic cleric Sheikh Mohammed Omran"; (d) "Complete coverage: London attacks." 


\begin{tabular}{|c|c|}
\hline Person & Role \\
\hline Mahmoud Abbas & $\overline{\text { Palestinian Authority President }}$ \\
\hline Kofi Annan & UN Secretary General \\
\hline Ian Blair & London Police Chief \\
\hline Tony Blair & Britain Prime Minister \\
\hline Bush & US President \\
\hline Charles Clarke & Britain Home Secretary \\
\hline Peter Clarke & London Police Anti-Terrorism Branch Head \\
\hline Saeb Erekat & Palestine negotiator \\
\hline Joseph Estrada & Phillipines deposed President \\
\hline John Howard & Australia Prime Minister \\
\hline Hasib Hussain & London Bomber \\
\hline Saddam Hussein & Ousted Iraq President \\
\hline Kim Jongil & North Korea Leader \\
\hline Kim Jong Il & North Korea Leader \\
\hline Laden & Al-Qaeda Leader \\
\hline Gloria Macapagal Arroyo & Phillipines President \\
\hline Ferdinand Marcos & Phillipines late President \\
\hline Scott McClellan & US White House President Secretary \\
\hline Shaul Mofaz & Israel Defense Minister \\
\hline Abu Musab & Al-Qaeda Iraq Member \\
\hline Pervez Musharraf & Pakistan President \\
\hline Richard Reid & Shoe Bomber London \\
\hline Condoleezza Rice & US Secretary of State \\
\hline Ariel Sharon & Israel Prime Minister \\
\hline Mohammad Sidique Khan & London Bomber \\
\hline Mohammed Sidique Khan & London Bomber \\
\hline Manmohan Singh & India Prime Minister \\
\hline Jack Straw & Britain Foreign Secretary \\
\hline Jalal Talabani & Iraq President \\
\hline Shahzad Tanweer & London Bomber \\
\hline Shehzad Tanweer & London Bomber \\
\hline Nasser Yousef & Palestinian Authority Interior Minister \\
\hline
\end{tabular}

Figure 3: The table shows the 32 names (in alphabetical order) that were selected for study over a period of about six weeks, from July 10, 2005 to August 26, 2005. The corresponding roles of the people are also shown. 
which a person's name appears. As outcome, the algorithm is able to assign probabilities to words (resp. people) as representing (resp. belonging to) different groups.

The first stage is implemented by applying Probabilistic Latent Semantic Analysis (PLSA) to the news corpus [8]. Due to lack of space, we briefly describe the PLSA process. Given a collection of $D$ documents $\left\{d_{i}\right\}$ spanning a vocabulary of $W$ words, PLSA models each word $w_{j}$ in a document as arising from a mixture model. The mixture components are multinomial hidden variables $z_{k}$ called aspects. A word can be generated by more than one aspect, and documents can thus be described by multiple aspects. Each $w_{j}$ is conditionally independent of the document $d_{i}$ it belongs to, given $z_{k}$. For $K$ aspects, the term-document joint probability is given by

$$
P\left(w_{j}, d_{i}\right)=P\left(d_{i}\right) \sum_{k=1}^{K} P\left(w_{j} \mid z_{k}\right) P\left(z_{k} \mid d_{i}\right) .
$$

With PLSA, a document $d_{i}$ is thus characterized by a $K$-dimensional vector corresponding to its distribution over aspects $P\left(z \mid d_{i}\right)$, or in other words, by a low-dimensional topic-based representation. The model is learned in an unsupervised way via Expectation-Maximization (EM). Details about PLSA can be found in [8].

The second stage of the algorithm finds groups of people based on two basic assumptions: (1) people who belong to the same group can often be described as spanning the same topics (i.e., they are likely to have similar topic distributions), and (2) people often belong to more than one group (i.e, they can be described by more than one typical topic distribution). Assuming $N$ people and $M$ groups, let $o_{n}$ and $g_{m}$ denote the $\mathrm{n}$-th person and $\mathrm{m}$-th group, respectively. The algorithm uses the person-document co-occurrence information and the PLSA document representation as input, and outputs an estimation of the probability of each person of belonging to each of the groups, $P\left(g_{m} \mid o_{n}\right)$. A modification of $K$-means clustering is first applied on the document corpus using the Hellinger-Bhattacharya distance between PLSA document representations. Specifically, the distance between two documents $d_{i}, d_{i *}$, represented by $P\left(z \mid d_{i}\right), P\left(z \mid d_{i^{*}}\right)$, respectively, is computed as

$$
\chi\left(d_{i}, d_{i^{*}}\right)=\left\{\sum_{k=1}^{K}\left\{\sqrt{P}\left(z_{k} \mid d_{i}\right)-\sqrt{P}\left(z_{k} \mid d_{i^{*}}\right)\right\}^{2}\right\}^{1 / 2} .
$$

After clustering the documents, the probabilities $P\left(g_{m} \mid o_{n}\right)$ are simply estimated as the fraction of documents in which person $o_{n}$ appears that have been assigned to the cluster $g_{m}$,

$$
P\left(g_{m} \mid o_{n}\right)=\frac{\sum_{i=1}^{D} 1\left[d_{i} \in g_{m}, d_{i} \circ o_{n}\right]}{\sum_{i=1}^{D} 1\left[d_{i} \circ o_{n}\right]},
$$

where $1[\cdot]$ is the indicator function, and $d_{i} \circ o_{n}$ denotes the binary person-in-document relation (i.e., person $o_{n}$ appears in document $d_{i}$ ). Additionally, we are interested in estimating group-based word distributions, to be able to characterize each group by its most representative words (in probabilistic terms). We get an estimate of the word distribution given a group $P\left(w_{j} \mid g_{m}\right)$ by

$$
P\left(w_{j} \mid g_{m}\right)=\sum_{k=1}^{K} P\left(w_{j} \mid z_{k}\right) P\left(z_{k} \mid g_{m}\right),
$$

where the conditional distribution of an aspect given a group is computed by marginalizing over all people,

$$
P\left(z_{k} \mid g_{m}\right)=\sum_{n=1}^{N} P\left(z_{k} \mid o_{n}, g_{m}\right) P\left(o_{n} \mid g_{m}\right),
$$
by

and the conditional distribution $P\left(z_{k} \mid o_{n}, g_{m}\right)$ is computed

$$
P\left(z_{k} \mid o_{n}, g_{m}\right)=\sum_{i=1}^{D} P\left(d_{i}\right) P\left(z_{k} \mid d_{i}\right) 1\left[d_{i} \circ o_{n}\right],
$$

so the sum only considers the set of articles in which person $o_{n}$ appears. As the outcome of the algorithm, each group $g_{m}$ represents a set of documents having a specific mixture distribution over aspects. Clustering over the aspect distributions provides a formal representation for distinct news issues, some of which might not have been explicitly captured by individual aspects. In this sense, note that estimating word distributions per group allows to characterize a group by words that, although might potentially belong to quite distinct topics, are nevertheless representative of the group's topic mixture.

\section{EXPERIMENTS}

\subsection{Data and parameter setting}

In experiments, news stories occurring in the six-week period described earlier, and containing at least one of the $N=32$ people described in Table 1, were identified and converted to bags-of words, using our vocabulary of $W=7413$ words. For learning the model from this data, documents which were too long or too short were removed, to reduce the effects of document size in the model. More specifically, we sorted each person's documents for each day, by their lengths. From this list, the first and third quartiles were determined, and only the news documents whose lenghts were in the inter-quartile range were kept for further processing. In the end, we were left with $D=20799$ documents. Regarding the parameters of the model, unless stated otherwise, we arbitrarily set the number of aspects to $K=7$, and the number of groups to $M=5$. The choice of these parameters has obviously an impact in the performance of the model, but we did not explore ways of setting them automatically.

\subsection{Group representation and membership}

In order to characterize each group, we represent it by the top 10 words (ranked by $P\left(w \mid g_{m}\right)$ ), and the top 10 people (ranked by $P\left(o_{n} \mid g_{m}\right)$ ). Figures 4 and 5 show the five groups obtained (all figures show the stemmed version of words). By inspection, one can see that the top ranked words clearly identify the news issue which each group represents. The top ranked people, per group, are expected to be the key players with respect to such news topic. From common knowledge about world news, one can see that our model indeed seems to do so. Group 1 corresponds to Palestine and Israel, Group 2 corresponds to Korea and nuclear issues, 
Group 3 corresponds to Iraq, Group 4 corresponds to London bomb. The words associated with Group 5 are somewhat ambiguous. However, the associated people indicate that it could correspond to Phillipines political turmoil. It is also interesting, and somewhat intuitive, that a couple of people appear in the top rank of multiple groups. This could either be a result of people's active participation in multiple world issues given their political roles (e.g. George Bush and Condoleeza Rice, who appear top-ranked in groups 13 ), or the occurrence of indirect references to certain people in several world topics (e.g. Osama bin Laden, who appears top-ranked in Group 3, but also in Groups 1 and 5).

In a second experiment, with the intention of observing the discriminating ability of individual aspects, we performed document clustering using only a few aspects at a time, instead of the full aspect distribution. To do this, the Hellinger-Bhattacharya distance was calculated considering only one or two relevant aspects, and groups were constructed as described in Section 3. In this case, we fixed the number of groups to $M=2$, to analyze if the algorithm could recover the group structure based on a "topic" vs. "non-topic" scenario. Figures 6 and 7 show the groups obtained when only the aspect probabilities corresponding to London bomb and IsraelPalestine, respectively, were used as features for clustering. In both cases, the probabilities $P(w \mid z)$ were used to identify the relevant aspects. For the case when only the London bomb-related aspects were used, the top 5 ranked people in the group related to this topic (as identified by the top ranked words) are indeed related to the subject, and also appeared in the group related to the London bombings obtained in our initial experiment (group 4 in Figure 4). Note also that there were some differences in specific rankings. The other group (shown also in Fig. 6) is quite mixed in topics and people. It is interesting to note that none of the people in the first group appeared as top-ranked in the second one. A similar trend can be observed for the case when only the Palestine-Israel-related aspects were used. Keeping in mind that that the number of aspects in PLSA in our experiments is rather small -so roughly speaking, each individual aspect relates to a specific news topic-, the result of these experiments highlight a fact: many news stories are mainly about a single topic, and thus people that make those news also relate to mainly one topic. An example of these situation could be the London terrorists. However, as our first experiment suggested, there are other people who naturally belong to different groups given the multiple events they are involved in.

Our method has the advantages of using content, rather than only binary co-occurrence information, to find groups, and of being able to assign probabilities of group membership to people. As an initial way of comparing our approach, in a third experiment we clustered people into groups using only co-occurrence data, i.e. names appearing on the same news article. Let $\mathcal{N}(n)$ and $\mathcal{N}\left(n^{*}\right)$ denote the number of news documents in which people $o_{n}$ and $o_{n *}$ occur, respectively, and $\mathcal{N}\left(n, n^{*}\right)$ denote the number of documents in which the two people cooccur. A pair-wise similarity measure between people $o_{n}$ and $o_{n^{*}}, s\left(o_{n}, o_{n^{*}}\right)$ was then defined as

$$
s\left(o_{n}, o_{n^{*}}\right)=\frac{\mathcal{N}\left(n, n^{*}\right)}{\min \left(\mathcal{N}(n), \mathcal{N}\left(n^{*}\right)\right)} .
$$

This similarity measure was then used as input to a spectral clustering algorithm to group people [13]. The algorithm constructs a pair-wise people similarity matrix. After matrix pre-processing, its spectrum (eigenvectors) is computed, the $M$ largest eigenvectors are stacked in columns in a new matrix, and the rows of this new matrix are normalized. Each row of this matrix constitutes a feature associated to each person. The rows of such matrix are then clustered using $K$-means (with $M$ clusters), and all people are labeled accordingly, which produces a hard partition of the list of people. Details of the spectral clustering algorithm can be found in [13]. In this experiment, we clustered people into $M=5$ groups, as before. The groups obtained are shown in Table 1. We can observe that, although some of the clusters are quite meaningful, e.g. group 3 relates to London bomb, group 4 to Israel-Palestine, and group 5 to Phillipines, the other groups turned out to be either very mixed or too small. Furthermore, some people that could naturally belong to several groups are assigned to only one of them by the spectral method. This is an inherent limitation of any hard-clustering approach.

\subsection{Studying people's topic evolution}

As an application of our framework, we looked at topic evolution with respect to a few multi-role key players in news. Specifically, for a given person, each word in their news articles was assigned to a group, based on the distribution $P\left(g_{m} \mid w\right)$. The temporal scale was divided into disjoint windows of five days, and the fraction of words corresponding to each person and assigned to each group was calculated over each time period. This fraction can be thought to represent the extent to which a particular person was involved in a certain news issue [18]. Figure 8 shows the results for six people. The legends in the graphs indicate the groups to which trends correspond to. We plot the trend for the four most prominent groups, namely Palestine, Iraq, London bomb, and North Korea, and identify them by the respective terms.

We now briefly discuss interesting trends with respect to individual people. In order to explain the trend, we refer to the fraction of words assigned to a certain group for a given person as the respective topic itself.

- George W. Bush - The topic London bomb is high and drops over time, which is expected, as it is a relatively short-lived issue. Topics Korea and Iraq fluctuate over time. Interestingly, we notice a high value of topic Palestine around the end, which is expected to be the approximate period of the Gaza pullout event.

- Condoleeza Rice - There is a relatively low value of the topic London bomb, all the time. However, we notice a peak in Palestine at the same time as for George W. Bush (approximate time of Gaza pullout). Topic Korea is particularly high, especially around the beginning and the end.

- Tony Blair - We notice a very high value of London bomb, fluctuating over time. This is an expected result. A peak in Palestine is noticed around the end, consistent with Condoleeza Rice and George Bush.

- Saddam Hussein - The topic Iraq dominates throughout which is again an expected result. The 


\begin{tabular}{|c|c|}
\hline Word & Prob. \\
\hline gaza & 0.0286 \\
palestinian & 0.0251 \\
israel & 0.0220 \\
israeli & 0.0218 \\
said & 0.0167 \\
settlement & 0.0122 \\
settl & 0.0103 \\
bank & 0.0093 \\
west & 0.0092 \\
pullout & 0.0076 \\
\hline Name & $\mathbf{P r o b}$ \\
\hline Nasser Yousef & 0.1813 \\
Saeb Erekat & 0.1810 \\
Mahmoud Abbas & 0.1727 \\
Ariel Sharon & 0.1721 \\
Shaul Mofaz & 0.1717 \\
Condoleezza Rice & 0.0611 \\
Bush & 0.0240 \\
Scott McClellan & 0.0114 \\
Laden & 0.0056 \\
Kofi Annan & 0.0046 \\
\hline
\end{tabular}

\begin{tabular}{|c|c|}
\hline Word & Prob. \\
\hline nuclear & 0.0236 \\
said & 0.0198 \\
north & 0.0195 \\
iran & 0.0187 \\
korea & 0.0169 \\
talk & 0.0163 \\
unit & 0.0080 \\
stat & 0.0071 \\
korean & 0.0070 \\
south & 0.0069 \\
\hline Name & Prob. \\
\hline Kim Jong II & 0.2426 \\
Kim Jongil & 0.2422 \\
Condoleezza Rice & 0.0904 \\
Scott McClellan & 0.0874 \\
Manmohan Singh & 0.0805 \\
Kofi Annan & 0.0702 \\
Bush & 0.0617 \\
John Howard & 0.0413 \\
Pervez Musharraf & 0.0303 \\
Jack Straw & 0.0178 \\
\hline
\end{tabular}

\begin{tabular}{|c|c|}
\hline Word & Prob. \\
\hline said & 0.0235 \\
iraq & 0.0190 \\
kill & 0.0162 \\
bomb & 0.0115 \\
attack & 0.0114 \\
iraqi & 0.0111 \\
sunni & 0.0090 \\
constitution & 0.0082 \\
baghdad & 0.0074 \\
police & 0.0073 \\
\hline Name & Prob. \\
\hline Jalal Talabani & 0.2506 \\
Abu Musab & 0.2264 \\
Saddam Hussein & 0.1986 \\
Scott McClellan & 0.0568 \\
Bush & 0.0566 \\
Laden & 0.0505 \\
Pervez Musharraf & 0.0352 \\
Condoleezza Rice & 0.0243 \\
Jack Straw & 0.0195 \\
Kofi Annan & 0.0184 \\
\hline
\end{tabular}

Figure 4: The figure shows groups 1, 2, and 3 (out of 5 groups), characterized by the words (ranked by $\left.P\left(w \mid g_{m}\right)\right)$ and people (ranked by $\left.P\left(o \mid g_{m}\right)\right)$.

\begin{tabular}{|c|c|}
\hline Word & Prob. \\
\hline bomb & 0.0288 \\
said & 0.0273 \\
london & 0.0262 \\
police & 0.0246 \\
attack & 0.0160 \\
suspect & 0.0116 \\
british & 0.0090 \\
arrest & 0.0083 \\
britain & 0.0071 \\
people & 0.0070 \\
\hline Name & Prob. \\
\hline Mohammed Sidique Khan & 0.0911 \\
Mohammad Sidique Khan & 0.0910 \\
Shahzad Tanweer & 0.0907 \\
Peter Clarke & 0.0907 \\
Hasib Hussain & 0.0903 \\
Richard Reid & 0.0894 \\
Shehzad Tanweer & 0.0894 \\
Ian Blair & 0.0871 \\
Charles Clarke & 0.0641 \\
Jack Straw & 0.0499 \\
\hline
\end{tabular}

\begin{tabular}{|c|c|}
\hline Word & Prob. \\
\hline said & 0.0169 \\
government & 0.0082 \\
minist & 0.0065 \\
lead & 0.0065 \\
people & 0.0060 \\
president & 0.0058 \\
country & 0.0056 \\
year & 0.0054 \\
world & 0.0053 \\
say & 0.0049 \\
\hline Name & Prob. \\
\hline Ferdinand Marcos & 0.1281 \\
Joseph Estrada & 0.1279 \\
Gloria Macapagal Arroyo & 0.1198 \\
John Howard & 0.0856 \\
Manmohan Singh & 0.0835 \\
Kofi Annan & 0.0794 \\
Tony Blair & 0.0593 \\
Laden & 0.0447 \\
Scott McClellan & 0.0425 \\
Pervez Musharraf & 0.0402 \\
\hline
\end{tabular}

Figure 5: The figure shows Groups 4 and 5 (out of 5 groups), characterized by the words (ranked by $P\left(w \mid g_{m}\right)$ ) and people (ranked by $P\left(o \mid g_{m}\right)$ ).

\begin{tabular}{|c|c|}
\hline Word & Prob. \\
\hline bomb & 0.0273 \\
said & 0.0268 \\
london & 0.0246 \\
police & 0.0232 \\
attack & 0.0154 \\
\hline Name & Prob. \\
\hline Richard Reid & 0.0833 \\
Mohammed Sidique Khan & 0.0833 \\
Mohammad Sidique Khan & 0.0833 \\
Shahzad Tanweer & 0.0831 \\
Shehzad Tanweer & 0.0830 \\
\hline
\end{tabular}

\begin{tabular}{|c|c|}
\hline Word & Prob. \\
\hline said & 0.0184 \\
gaza & 0.0081 \\
palestinian & 0.0071 \\
israel & 0.0063 \\
israeli & 0.0062 \\
\hline Name & Prob. \\
\hline Kim Jong II & 0.0498 \\
Jalal Talabani & 0.0498 \\
Ferdinand Marcos & 0.0498 \\
Kim Jongil & 0.0497 \\
Ariel Sharon & 0.0495 \\
\hline
\end{tabular}

Figure 6: The figure shows the two groups obtained, characterized by the words (ranked by $\left.P\left(w \mid g_{m}\right)\right)$ and people (ranked by $P\left(o \mid g_{m}\right)$ ), using a constrained distance measure for clustering, where only aspects related to London bomb were used in the distance. 


\begin{tabular}{|c|c|}
\hline Word & Prob. \\
\hline gaza & 0.0282 \\
palestinian & 0.0247 \\
israel & 0.0217 \\
israeli & 0.0215 \\
said & 0.0168 \\
\hline Name & Prob. \\
\hline Saeb Erekat & 0.1779 \\
Nasser Yousef & 0.1776 \\
Ariel Sharon & 0.1726 \\
Shaul Mofaz & 0.1722 \\
Mahmoud Abbas & 0.1711 \\
\hline
\end{tabular}

\begin{tabular}{|c|c|}
\hline Word & Prob. \\
\hline said & 0.0226 \\
bomb & 0.0147 \\
police & 0.0120 \\
london & 0.0116 \\
attack & 0.0092 \\
\hline Name & Prob. \\
\hline Shehzad Tanweer & 0.0379 \\
Shahzad Tanweer & 0.0379 \\
Richard Reid & 0.0379 \\
Peter Clarke & 0.0379 \\
Mohammed Sidique Khan & 0.0379 \\
\hline
\end{tabular}

Figure 7: The figure shows the two groups obtained, characterized by the words (ranked by $\left.P\left(w \mid g_{m}\right)\right)$ and people (ranked by $P\left(o \mid g_{m}\right)$ ), using a constrained distance measure for clustering, where only aspects related to Israel-Palestine were used in the distance.

\begin{tabular}{|c|c|c|c|c|}
\hline Group 1 & Group 2 & Group 3 & Group 4 & Group 5 \\
\hline Abu Musab & Joseph Estrada & Charles Clarke & Ariel Sharon & Ferdinand Marcos \\
Bush & & Hasib Hussain & Condoleezza Rice & Gloria Macapagal Arroyo \\
Jalal Talabani & & Ian Blair & Mahmoud Abbas & Nasser Yousef \\
John Howard & & Jack Straw & Saeb Erekat & \\
Kim Jongil & & Mohammad Sidique Khan & Shaul Mofaz & \\
Kim Jong Il & & Mohammed Sidique Khan & \\
Kofi Annan & & Pervez Musharraf & \\
Laden & & Peter Clarke & \\
Manmohan Singh & & Richard Reid & \\
Saddam Hussein & & Shahzad Tanweer & & \\
Scott McClellan & & Shehzad Tanweer & & \\
& & Tony Blair & \\
\hline
\end{tabular}

Table 1: Groups of people obtained using a spectral clustering method and co-occurrence-only data.

remaining topics do have small values throughout. This could be primarily because of the inherent similarity in the topics, such as concerns about security, nuclear threats, terrorism, and hence a somewhat similar use of language.

- Pervez Musharraf - The topic London bomb is high and drops over time. Topic Iraq fluctuates over time. A small peak in Palestine is noticed towards the end. A particularly high value of Korea is seen. Although Mr. Musharraf has no direct relation to the subject, this peak could again be explained as the result of similar use of language and similar concerns across the topics.

- Osama bin Laden - The topic London bomb is high and drops over time. A peak in Palestine is noticed towards the end. The rest of the topics fluctuate over time.

\subsection{Classification of new documents}

For each group, a representation was obtained as the centroid of all documents' aspect distributions $P(z \mid d)$ which form the particular group, and computed during training. A classifier for new documents containing at least one of the people listed in Table 3 was set up as follows. Each group was first identified by the $\mathcal{N}$ top ranked people, where people were ranked based on the probabilities $P\left(o_{n} \mid g_{m}\right)$. Then, for a new document $d_{\text {new }}$, a group $g_{m^{n e w}}$ was first assigned using nearest neighbor criterion. The distance used here was the Hellinger-Bhattacharya distance, as explained earlier. During classification, each occurrence of a person was treated as an instance of the document. For the new document $d_{\text {new }}$, classification to group $g_{m^{n e w}}$ was deemed correct if the corresponding person $o_{n}$ new was one of the $\mathcal{N}$

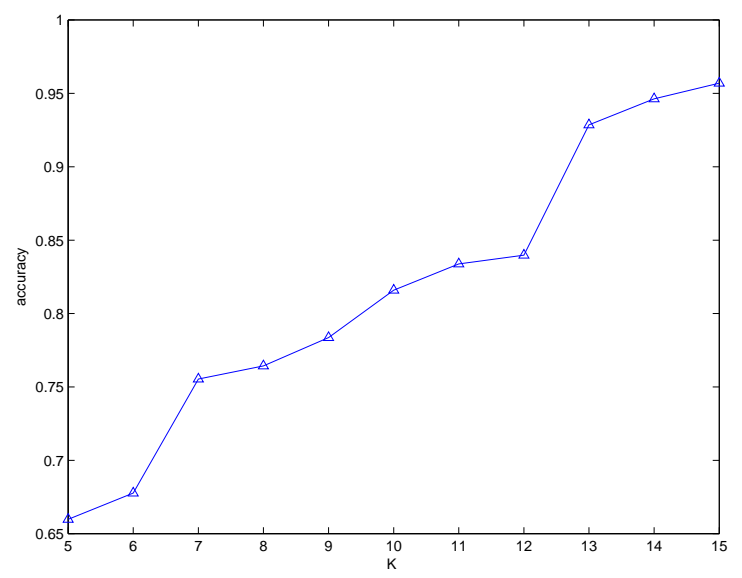

Figure 9: Classification performance of the algorithm for different values of parameter $\mathcal{N}$, i.e., the number of representatives each group $g_{m}$ is represented by.

representatives of $g_{m^{n e w}}$. The classification performance for different values of $\mathcal{N}$ is shown in Figure 9. The total number of documents used for testing purposes was 3157 (the set is obviously disjoint from the documents used for training). We observe that this simple approach can correctly predict documents as containing the correct person -based on the documents' content- in more than $80 \%$ of the cases when $\mathcal{N} \geq 10$. This is an interesting result given that the method is fully unsupervised, but would of course need to be validated against a standard supervised approach in order to assess its comparative performance. 


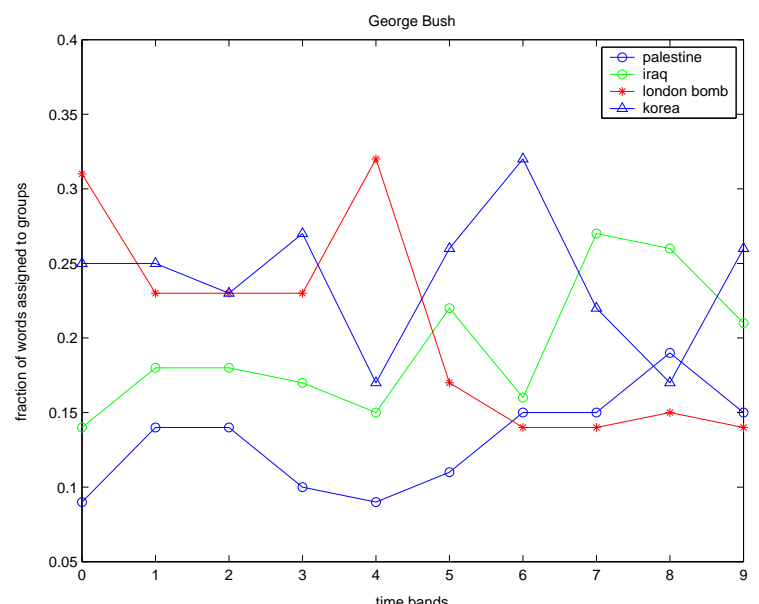

(a)

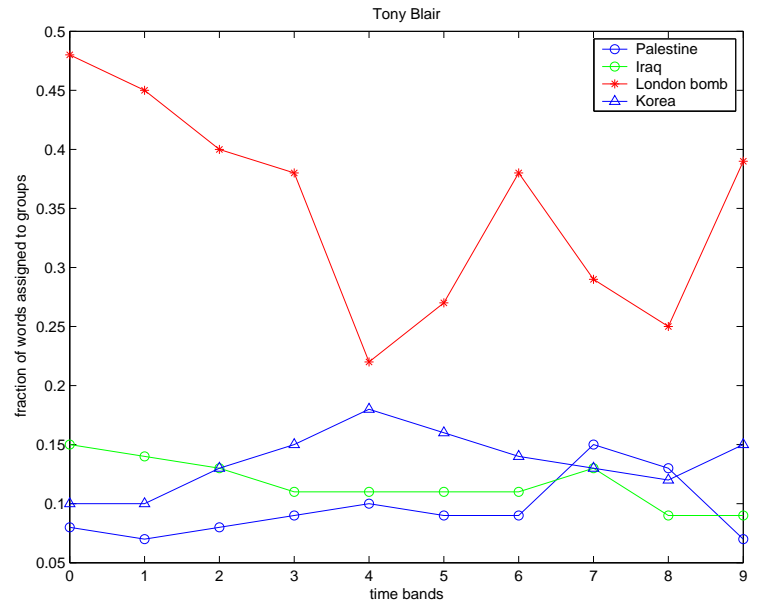

(c)

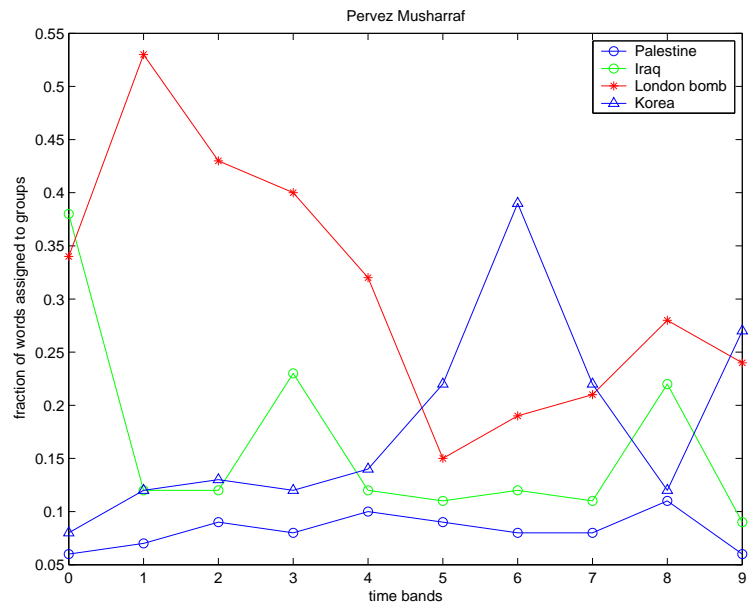

(e)

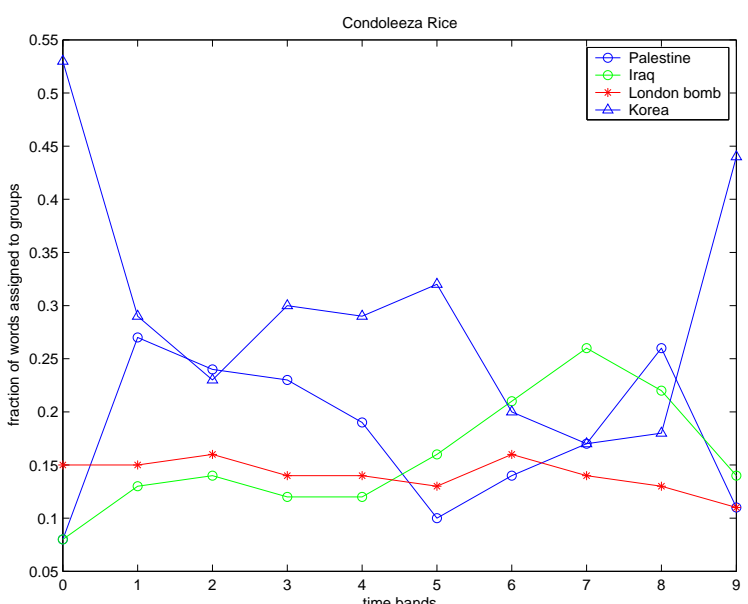

(b)

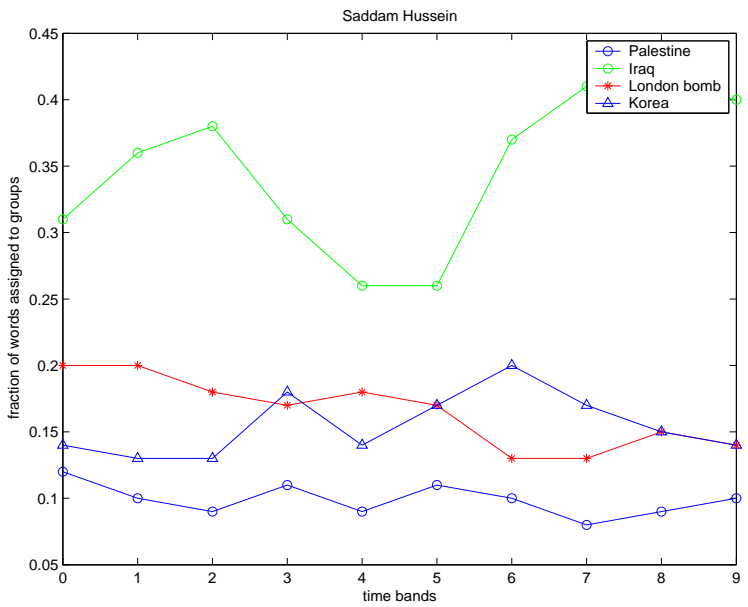

(d)

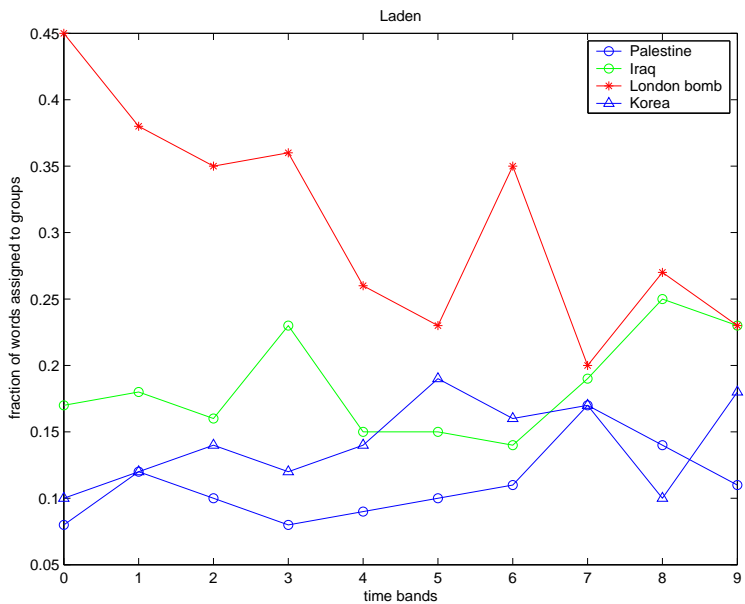

(f)

Figure 8: The figure shows topic evolution with respect to certain people, over a period of about six weeks. In each graph, the $X$ axis represents the time period, and the $Y$ axis represents fraction of words per group. The people are (a) George Bush, (b) Condoleeza Rice, (c) Tony Blair, (d) Saddam Hussain, (e) Pervez Musharraf, and (f) Osama bin Laden. 


\section{DISCUSSION AND CONCLUSION}

We studied the problem of social network discovery from Web news data. Groups were constructed based on the representation of news stories as mixtures of aspects using probabilistic latent semantic analysis-, and a simple probabilistic framework to associate people with groups was proposed. This was used to quantify the involvement of people in different groups over time. The study of both the discovered groups and the evolution of topics was coherent with our common knowledge about world events, but is however more difficult to assess objectively. A systematic objective evaluation of our approach is clearly a non-trivial issue that requires further work. Such an evaluation would include the effect of varying the parameters that were kept as part of this study. The problem of scalability to larger number of people and amount of news also needs to be studied.

One potential extension of this work could be to incorporate image information, leading to the construction of a social network from multimedia data. We could consider the images embedded in Web news articles and design a system to learn a social network between commonly occurring people from image data. Automatic face detection/recognition methods would be necessary for this purpose. This model could then be integrated with our current work, which uses only text. It would indeed be interesting to study the coherence of social networks learned using text and image data.

However, there are a few challenges involved in this direction. In the first place, automatic image collector programs which search the Web often bring a very large number of unrelated images, e.g. logos, icons, and advertisements. In order to perform analysis, an effective (and maybe semiautomatic) algorithm for pruning this dataset would likely be required. Issues to evaluate include whether the number of relevant images collected in this fashion would be sufficient to learn a meaningful social network, and whether visual processing algorithms would be robust enough for reliable person identification. In the second place, relevant images occurring within news stories often contain no people at all. Finally, certain people occurring frequently in news text could be seldom depicted in pictures. All these issues have to be considered for future work in this domain.

\section{ACKNOWLEDGEMENT}

This work was supported by the Swiss NCCR on Interactive Multimodal Information Management (IM2). The authors would like to thank Florent Monay and Dong Zhang for insightful discussions during this work.

\section{REFERENCES}

[1] R. Baeza-Yates and B. Ribeiro-Neto, "Modern Information Retrieval," ACM Press, 1999.

[2] A. L. Barabasi, "Linked: The New Science of Networks," Perseus, 2002.

[3] A. L. Barabasi, R. Albert, H. Jeong, "Scale-free Characteristics of Random Networks: the Topology of the World Wide Web," Physica, 281, 69-77, 2000.

[4] T. Berg, A. Berg, J. Edwards, M. Maire, R. White, Y.-W. Teh, E.L. Miller, and D. Forsyth, "Faces and Names in the News," Proc. IEEE Int. Conf. on
Computer Vision and Pattern Recognition (CVPR), 2004.

[5] D. Blei, A. Ng, and M. I. Jordan, "Latent Dirichlet Allocation," Journal of Machine Learning Research, vol. 3, 993-1022, 2003.

[6] T. Choudhury and A. Pentland, "Sensing and Modeling Human Networks using the Sociometer," Proc. Int. Conf. on Wearable Computing, 2003.

[7] J. Golbeck, B. Parsia, and J. Hendler, "Trust Networks on the Semantic Web," Lecture Notes in Computer Science, 238-249, 2003.

[8] T. Hofmann, "Unsupervised Learning by Probabilistic Latent Semantic Analysis," Machine Learning, vol. 42, 177-196, 2001.

[9] B. Klimt and Y. Tang, "The Enron Corpus: A New Dataset for Email Classification Research" Proc. European Conf. on Machine Learning, 2004.

[10] D. Lewis, "Reuters-21578 Test Categorization Test Collection," http://kdd.ics.uci.edu/databases/reuters21578.html, 1997.

[11] A. McCallum, A. Corrada-Emmanuel, and X. Wang, "Topic and Role Discovery in Social Networks," Proc. Int. Joint Conf. on Artificial Intelligence, 2005.

[12] M. E. J. Newman, "Coauthorship Networks and Patterns of Scientific Collaboration," Proc. National Academy of Sciences, 2004.

[13] A. Ng, M. I. Jordan, and Y. Weiss, "On Spectral Clustering: Analysis and an Algorithm," Proc. Neural Information Processing, 2001.

[14] K. Nowicki and T. A. B. Snijders, "Estimation and Prediction for Stochastic Blockstructures," Journal of American Statistical Association, vol. 96, 1077-1087, 2001.

[15] A. Ozgur and H. Bingol, "Social Networks of Co-occurence in News Articles," Lecture Notes in Computer Science, 3280, 688-695, 2004.

[16] A. Pentland, "Socially Aware Computation and Communication," IEEE Computer, vol. 38, 33-40, 2005.

[17] J. Preece, "Online Communities: Designing Usability and Supporting Sociability," John Wiley \& Sons, 2000.

[18] M. Rosen-Zvi, T. Griffiths, M. Steyvers, and P. Smyth, "The Author Topic Model for Authors and Documents," Proc. 20th conference on Uncertainty in Artificial Intelligence, 2004.

[19] N. A. Van House and M. Davis, "The Social Life of Cameraphone Images,". Proc. Pervasive Image Capture and Sharing Workshop at the Seventh Int. Conf. on Ubiquitous Computing, 2005.

[20] X. Wang, N. Mohanty, and A. McCallum, "Group and Topic Discovery from Relations and Text," Proc. KDD Workshop on Link Discovery: Issues, Approaches and Applications, 2005.

[21] S. Wasserman and K. Faust, "Social Network Analysis: Methods and Applications," Cambridge University Press, 1994. 